\title{
Koiné y aticismo en el tratado de Galeno Sobre los procedimientos anatómicos
}

Koine and Atticism in the Galenic Treatise

On Anatomical Procedures

\author{
Jordi REDONDO \\ Universitat de València \\ jordi.redondo@valencia.edu
}

\begin{abstract}
RESUMEN: El tratado Sobre los procedimientos anatómicos ofrece un extenso corpus para el estudio de la lengua literaria de Galeno. La naturaleza de ésta ha sido alineada tanto en las filas de un aticismo moderado como en las del antiaticismo. Frente a esta segunda opción, que abunda en la definición de la obra desde una óptica que excluye todo fin literario de la misma, los frecuentes recursos retóricos muestran un buen conocimiento de la prosa artística por parte de nuestro autor. Ahora bien, el análisis de la lengua demuestra que está basada en la koiné no literaria.
\end{abstract}

ABSTRACT: The treatise entitled On anatomical procedures displays an extensive corpus in order to analyze the Galenic literary language. Its patterns have been diversely labelled, either as belonging to the ranks of a moderate Atticism or to the ranks of Antiatticism. Again this second option, which speaks for the understanding of the treatise beyond any literary intention, the use of many different rhetorical devices shows a good knowledge of artistical prose by our author. Nevertheless, the analysis of language proves that it is based on not-literary Koine.

PALABRAS Clave: Aticismo; koiné; retórica; prosa científica.

KeYwords: Atticism; Koine; Rhetoric; Scientific Prose.

RECIBIDO: 27 de diciembre de 2016 - ACEPTADO: 1 de abril de 2017.

DOI: 10.19130/iifl.nt.2017.35.1.754

La lengua literaria de Galeno se ha descrito como una lexis sencilla, que se encuadraría en los usos propios de la koiné, aunque con ciertas concesiones al aticismo. La tradición investigadora sobre nuestro autor ha otorgado un ilimitado crédito a sus numerosos testimonios de reprobación del aticismo. ${ }^{1}$ De ahí, muy probablemente, que se haya creado la imagen de un Galeno antiaticista. De manera más matizada, Horrocks lo considera el representante de una oposición práctica, no necesariamente

${ }^{1}$ Véase el exhaustivo elenco recopilado por Herbst (1911), si bien cabe apuntar que esta obra ha condicionado en exceso la investigación posterior. 
teórica, a las exigencias del aticismo. ${ }^{2}$ Más alejada de la realidad parece la opinión de Goldhill, que define el griego de Galeno como un mero producto literario. ${ }^{3}$ La mejor aproximación al tema es el reciente estudio de Vela sobre De antidotis, cuyas conclusiones sitúan a nuestro autor en la koiné literaria. ${ }^{4}$ A esta lengua, en la que unos han visto un fondo predominante de corte clásico, y otros uno basado en la koiné, Palm la llama hellenistische Normalprosa, ${ }^{5}$ Rydbeck Zwischenschichtsprosa, ${ }^{6} \mathrm{y}$ Horrocks artistically 'developed' Koine. ${ }^{7}$

Muy oportunamente, Swain ha puesto de relieve la comodidad de Galeno ante las exigencias del aticismo, que veía hasta cierto punto intrascendentes en su caso, ya que la lengua técnica de la medicina carecía de un referente ático al estar el Corpus Hippocraticum compuesto en

${ }^{2}$ Horrocks $2010^{2}$, pp. 136-137: "Despite the fact that Atticism tended to smother natural invention by encouraging a preoccupation with linguistic form and institutionalizing a state of mind that equated a surface dressing of 'hallmarked' items with learning and good taste, the hold of the movement in educated circles was such that those who failed to display the expected knowledge of approved grammar and diction forfeited all prospect of serious consideration. Only writers of scientific prose, such as the Pergamene physician Galen (AD 129-99), were in a position to reject its demands (in part) in the interests of clarity and precision".

${ }^{3}$ Goldhill 2002, p. 90: "Most elite writing, certainly most intellectual activity, including rhetorical performance, was conducted in versions of Attic Greek of the Classical period. Two obvious and important exceptions may be due to genre: a doctor such as Galen, and the philosopher/emperor Marcus Aurelius, each write in a sophisticated literary Greek which does not closely imitate Classical Attic, though it is still removed from Koine".

${ }^{4}$ Vela Tejada 2009, p. 59: “Creemos, en definitiva, que nos hallamos ante un tipo de lengua de altas pretensiones literarias que no deja de estar firmemente anclada en el tipo

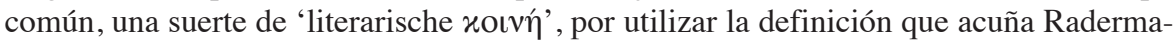
cher, diferenciada de la "mündliche xoเví'".

${ }^{5}$ Palm 1955, p. 206: "es scheint mir praktischer, den Terminus Koine nur von der nachklassischen Volkssprache zu gebrauchen und die Sprachen, die ein Philon von Byzanz, ein Apollonios von Perge, ein Polybios usw. verwendet haben, als hellenistische Normalprosa zu bezeichnen".

${ }^{6}$ Rydbeck 1967, pp. 189-192 y 200-203.

${ }^{7}$ Horrocks $2010^{2}$, p. 96: "Many prose authors of both the Hellenistic and Roman imperial times [...] used a literary language distinct from 'Classical' Attic of authors of the $5^{\text {th }}$ and $4^{\text {th }}$ centuries $\mathrm{BC}$ which might fairly be regarded as an artistically developed version of the Koine employed by the Hellenistic/Roman bureaucracies. Whether we choose to see this as a diluted variety of Classical Attic or to refer to it rather as a a 'literary' version of the Koine is ultimately no more than a terminological issue". 
una lengua de base jonia ${ }^{8}$ - otra cosa es que dicha base jonia se halle entreverada con numerosas muestras de la influencia del ático, como bien demostró López Eire-. ${ }^{9}$ La valoración del aticismo por parte de Galeno mostraría, según Swain, una actitud ambivalente, a medias entre la aceptación y el rechazo. ${ }^{10}$ Por consiguiente, no habrá de extrañar que nuestro autor patentizara su conformidad con determinados planteamientos del aticismo. ${ }^{11}$ En otras palabras, en su afán por establecer un modelo de lengua científica, Galeno, que partía de la base del griego hablado por personas con una cierta formación, no excluiría algunas soluciones tomadas de la tradición literaria de la prosa ática. Por él mismo sabemos que reconocía en su lengua, se supone tanto la hablada como la escrita, el griego de la época, la koiné, a la vez que reivindicaba la pureza de su idiolecto, forjado en las enseñanzas de su padre. ${ }^{12}$ Debemos al propio médico de Pérgamo, autor de un extremo rigor intelectual y una amplia y profunda formación, una idea clara de lo que para él significaba el buen uso en materia lingüística. Su modelo, el de la ciencia médica, llega a personalizarse en la figura del maestro Hipócrates, cuya lengua define mediante los conceptos de la claridad, la corrección y la brevedad, esto

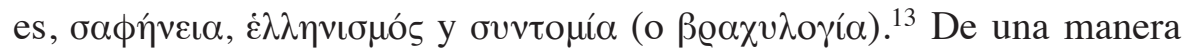
que no ha de sorprendernos, los conceptos clave para Galeno son los mismos que emplea Dionisio de Halicarnaso para definir la lengua de Tucídides. ${ }^{14}$

Por otra parte, si bien la lengua de la medicina contaba con una poderosa y bien acreditada tradición jonia, Galeno tenía su opinión per-

${ }^{8}$ Swain 1996, pp. 56-57.

${ }^{9}$ López Eire 1984.

${ }^{10}$ Swain 1996, p. 59: “Galen's attitude towards atticism is, then, quite complex. He was strongly drawn to the idea of purity in language (where Greekness was to be maintained to all costs), he had been reared on the canonical texts, and thus well equipped to write atticizing Greek. But he rejected this course because he saw no reason to use any other form of Greek than the excellent language he had learnt as a child, the general language of communication".

${ }^{11}$ Swain, op. cit., pp. 61-62.

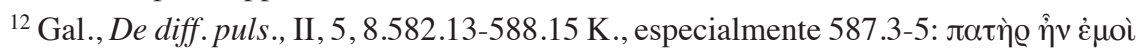

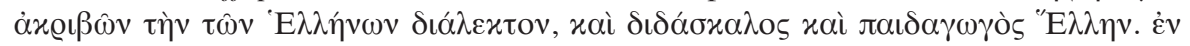

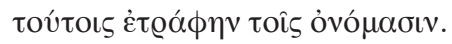

${ }^{13}$ Manetti 2003, p. 173.

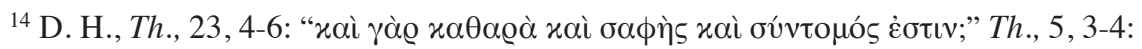

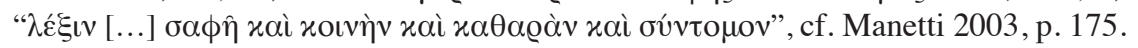


sonal en lo que hace a la obra de Hipócrates. Sin duda la pérdida del tratado galénico Sobre la lengua de Hipócrates nos habría aportado un documento precioso acerca del particular, pero al menos contamos con un brevísimo extracto del mismo. En su comentario a Sobre las fracturas,

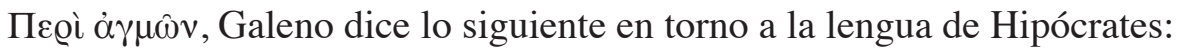

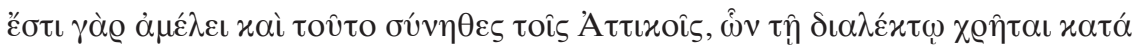

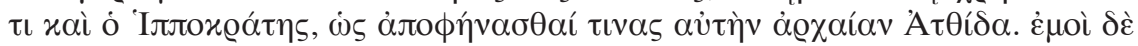

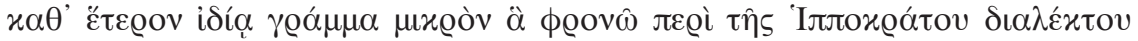
$\delta \varepsilon \delta \dot{\eta} \lambda \omega \tau \alpha \iota, x \tau \lambda .{ }^{15}$

A nuestro maestro Antonio López Eire oímos muchas veces que la pureza no es de este mundo, al menos en lo que a las lenguas respecta. La koiné pura de Galeno se antoja una ilusión de mal maridar con la realidad de nuestros textos, único espacio en el que ha de trabajar el filólogo. Y para ilustrar en qué emplea la lengua de Galeno a un tiempo soluciones aticistas y no aticistas comenzaremos por ver algo de su estilo, en el que hallamos también recursos retóricos, literarios, al lado de otros medios de expresión que derivan directamente de la lengua hablada en contextos que nada tienen que ver con la literatura. Por lo pronto, y desde el punto de vista metodológico, parece oportuno rechazar un planteamiento muy común, el de considerar al médico pergamense un autor técnico, desprovisto de todo interés por la creación literaria y que tan sólo habría recurrido a aquellos elementos propios del estilo de la prosa científica. ${ }^{16}$ Justo al contrario, las calidades estilísticas de la prosa galénica han llamado la atención de todo aquel que la ha frecuentado. Se ha puesto de relieve, por ejemplo, cómo la tendencia de Galeno a embellecer la prosa de sus tratados se trasluce en sus numerosas citas poéticas. ${ }^{17}$ Este ornato de la lexis galénica abarca también un buen número de recursos estilísticos. ${ }^{18}$ Más aún, Durling apunta la idea de que el interés

${ }^{15}$ Gal., In Hipp. Fract., 18/2.1.1.1.322.10-14. Todas las traducciones, allí donde hemos creído oportuno incluirlas, son del autor.

${ }^{16}$ Sobre los planteamientos literarios de Galeno en el horizonte cultural de la época, cf. Kollesch 1981; von Staden 1997; sobre las características formales de la prosa médica, cf. Kollesch 1992; van der Eijk 1997; Wittern-Sterzel 1998.

${ }^{17}$ De Lacy 1966; Vogt 2005.

18 Totelin 2012. 
de Galeno por una lexis más elaborada y rica crece con el tiempo. ${ }^{19}$ Pues bien, en el extenso tratado titulado Sobre los procedimientos anatómicos hemos notado el empleo de recursos tales como la aliteración, el políptoton, la duplicatio, la litote, la paronomasia y la uariatio. Veamos en primer lugar un ejemplo de aliteración:

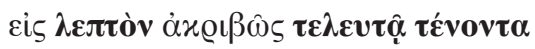

(II, 5.6.508.1).

Tampoco el políptoton es ajeno a nuestro autor, aunque su utilización no resulte abundante al tratarse de un recurso muy llamativo. En el presente tratado puede leerse un ejemplo como el siguiente:

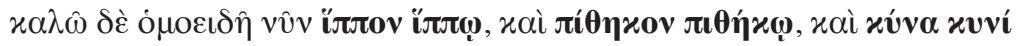

El empleo de la duplicatio no es aquí propiamente una figura retórica, sino un rasgo propio de la lengua científica en su afán de alcanzar una expresión precisa:

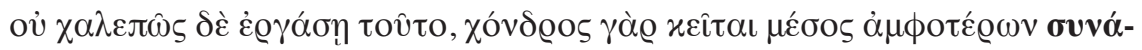

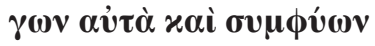

(II, 2.6.314.1-3).

Veamos un segundo ejemplo, que roza lo que los antiguos denominaban hendiadys, esto suponía la sinonimia de los dos conceptos expresados:

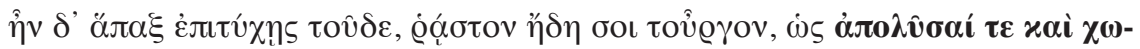

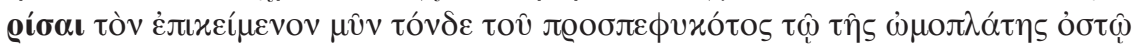
(II, 5.2.487.11-14).

Tampoco es ajeno nuestro autor al uso de la litote:

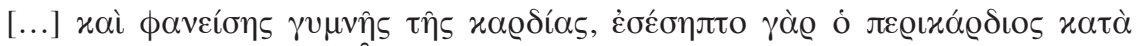

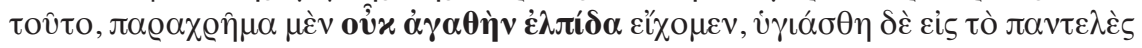

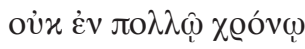

(II, 7.13.633.13-16).

${ }^{19}$ Durling 1988, p. 183: "I suspect that a chronological study of Galen's particles would show a development from simplicity to mannerism". 
En el siguiente ejemplo, Galeno emplea el mismo término, $\sigma \chi 0 \lambda \eta$, con dos valores diferentes, en el primer caso como substantivo con la acepción de estudio, derivada del valor originario de ocio, tiempo libre, y en el segundo como adverbio con la acepción a buenas horas, para largo, aplicada a aquello para lo que hay que tomarse mucho tiempo. Se trata, por lo tanto, de la figura de la paronomasia o annominatio, basada en la repetición de conceptos formalmente similares, pero de significado opuesto o diverso, ya que en este pasaje el primer empleo del término es positivo, y el segundo negativo:

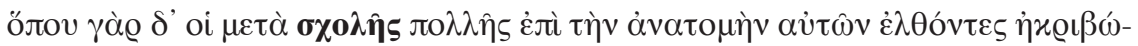

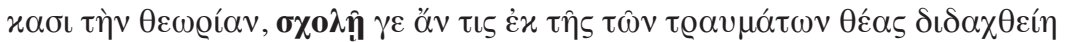

(II, 3.289).

Allí donde ni siquiera los que se aplican a la disección con gran estudio han llegado a precisar su teoría, a buenas horas iba alguien a aprender de la mera contemplación de las heridas.

En otro lugar, Galeno se permite, con indudable ironía, una frase que reproduce el estilo de la sofística, con hasta tres substantivos abstractos y el empleo de la figura etimológica:

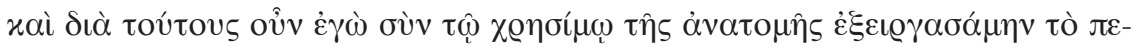

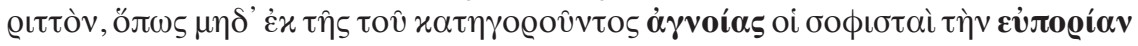

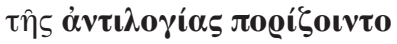

(II, 2.2.285.12-15).

Especial mención merece el uso de la uariatio. Con toda razón reclama López Férez atención hacia esta figura, ${ }^{20}$ de la que hay ejemplos tanto a nivel morfológico como sintáctico, léxico y estilístico:

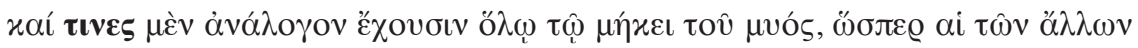

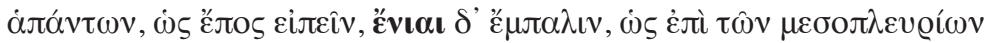

(II, 8.5.680.4-7).

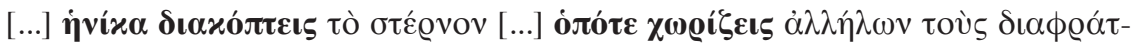

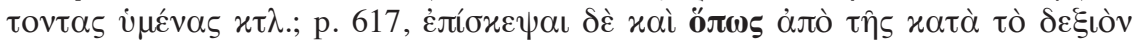

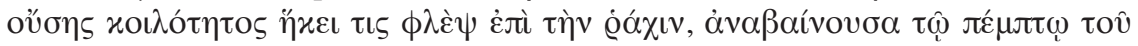

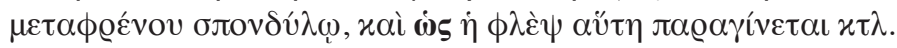

(II, 7.2.593.12-14).

${ }^{20}$ López Férez 1999, p. 346. 


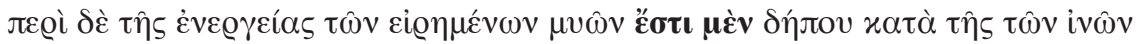

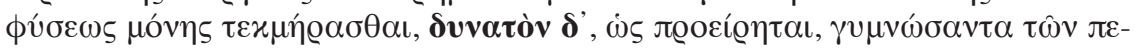

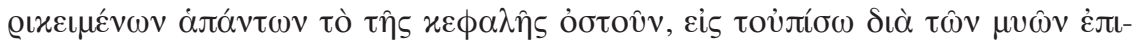

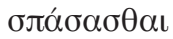

(II, 4.6.453.9-12).

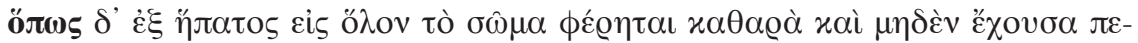

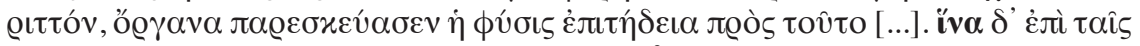

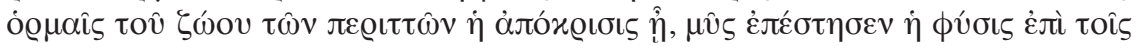

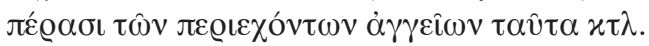

$$
\text { (II, 6.2.543.2-5 y 14-17). }
$$

Mayor valor aún tienen aquellos pasajes donde aparecen diversas figuras retóricas y estilísticas. Véase el siguiente ejemplo, en el que se combinan la uariatio en el empleo de las conjunciones completivas y la anadiplosis entre las dos frases:

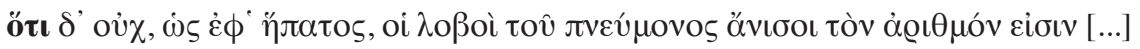

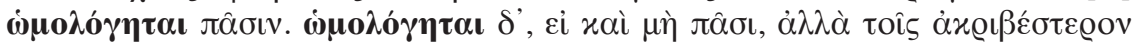

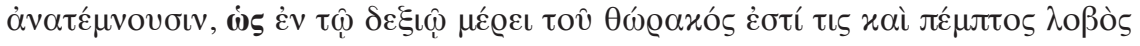
$\mu \varkappa \varrho o ́ s ~ x \tau \lambda$.

(II, 7.11.625.12-18).

Sirva como paradigma del interés de Galeno por elevar el estilo de su lengua literaria la frase siguiente:

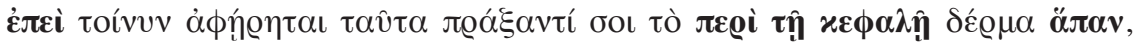

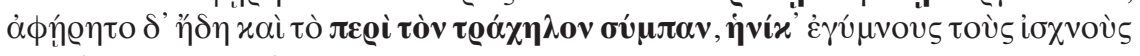

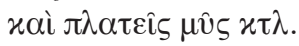

(II, 4.6.445.4-7).

Galeno construye sendas oraciones subordinadas temporales por me-

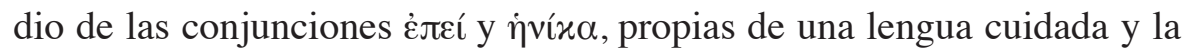
segunda de ellas de innegable carácter literario, a la vez que utiliza en uariatio los adjetivos cuantitativos ö $\alpha \alpha v$ у бú $\mu \pi \alpha v$, lo mismo ocurre con

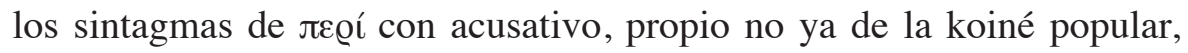
sino de lo que podríamos denominar el griego estándar, y лع@í con dativo, que es en cambio un sintagma perteneciente a la lengua de la poesía y casi del todo ausente, por tanto, de los géneros prosísticos. ${ }^{21}$ En resu-

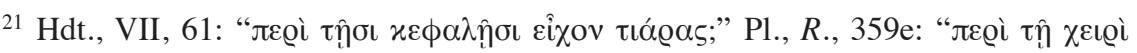

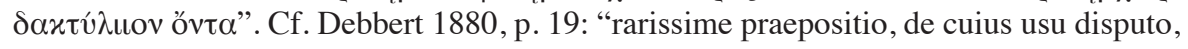


midas cuentas, tenemos aquí un triple ejemplo de uariatio, ya que a la

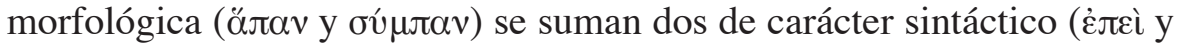

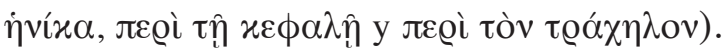

Una buena piedra de toque para comprobar el grado de las aspiraciones literarias de Galeno se halla en el empleo de verbos compuestos de los tipos $\tau \varrho เ \pi \lambda \hat{\alpha}$ y $\tau \varepsilon \tau \varrho \alpha \tau \lambda \hat{\alpha}$, entre los que se cuentan casos como,

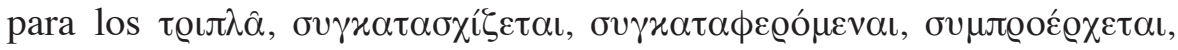

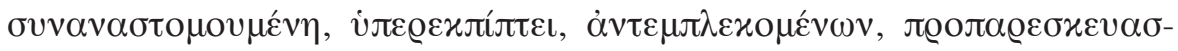

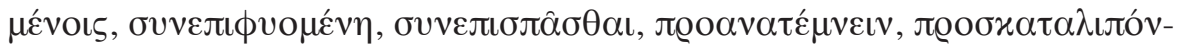

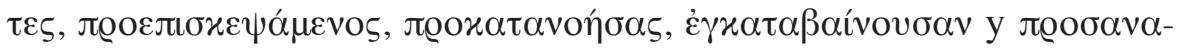

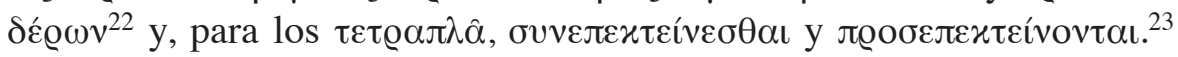

El propósito del presente artículo no es el ofrecer un análisis del estilo de Galeno, sino el comprobar cómo en su prosa coexisten koiné y aticismo. Luego de haber comprobado que en la lengua literaria del tratado que nos ocupa se registran abundantes y variados recursos de carácter literario será más fácil evaluar la naturaleza y la función de las soluciones aticistas y de koiné empleadas por nuestro autor.

Entre las soluciones aticistas de la prosa galénica se cuenta la $\tau \tau$, indicativa por sí misma del modelo literario de la misma. En los Proce-

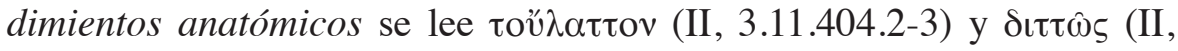

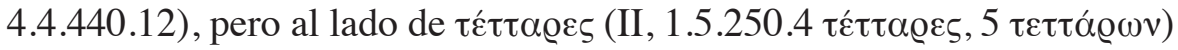

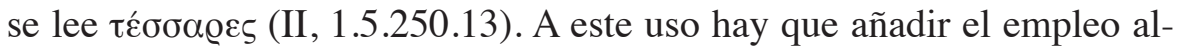

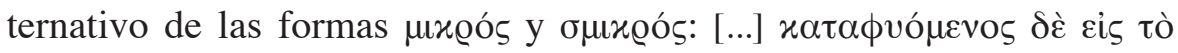

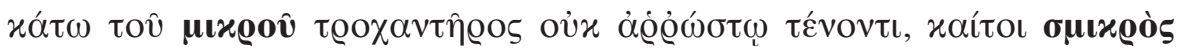
aủtòs őv (II, 2.6.307.15-17). En general se aprecian usos mayoritariamente aticistas, en términos de Vela Tejada. ${ }^{24}$

El aticismo recomendaba la utilización del dual, que en efecto se halla en la prosa de Galeno. Pero se trata de un empleo, al menos en un tratado de índole técnica, como lo es Sobre los procedimientos anatómicos, que no pasa de ser testimonial. Hay un único uso de dual nominal,

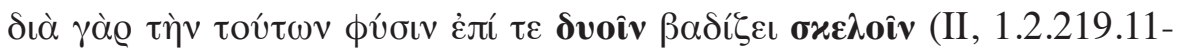

locali potestate praedita prosa in oratione invenitur. Neque ullum exemplum Thucydideum invenio".

22 Se hallan, respectivamente, en las pp. 375, 376, 377, 382, 428, 434, 441, 446, 458, 504, 531, 540, 606, 709 y 719 de la edición de Kühn.

${ }^{23}$ Cf. pp. 507, 522, 562 y 700, y 655 de la edición de Kühn.

${ }^{24}$ Vela Tejada 2009, pp. 43-44. 


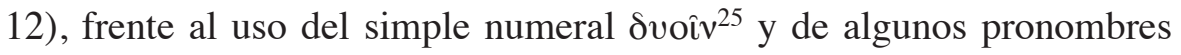
como $\dot{\alpha} \lambda \lambda \hat{n} \lambda$ oıv (II, 2.4.295.16), $\dot{\alpha} \mu \phi$ oîv (II, 5.6.510.11; II, 6.2.543.8 y II, 8.7.686.7) y aủंoîv (II, 6.1.540.7). Nótese también, junto a la total ausencia del dual verbal, que los ejemplos citados son siempre de los casos oblicuos.

La morfología verbal presenta la regularización paradigmática propia

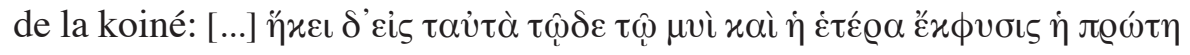

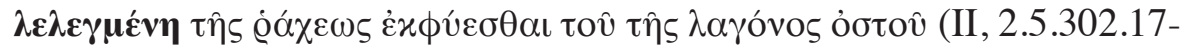

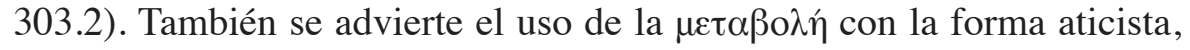

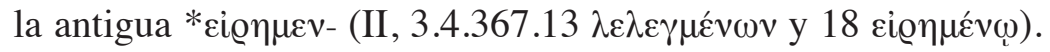

Tampoco la categoría del pronombre se libra de nivelaciones paradig-

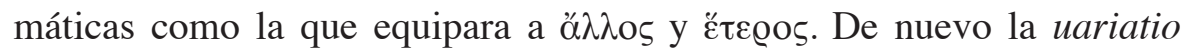
ayuda a demostrar con claridad cómo para Galeno ambas posibilidades

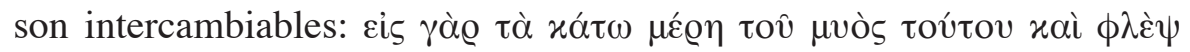

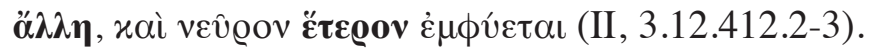

La sintaxis acusa también la influencia de la koiné no literaria. Así sucede con las oraciones exhortativas y prohibitivas introducidas por la negación $\mu \eta \dot{\pi} \omega \varsigma$ :

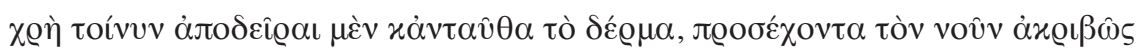

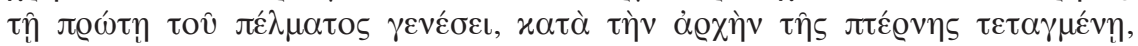

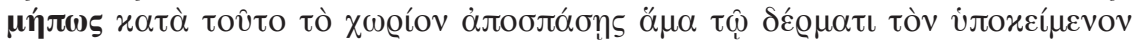

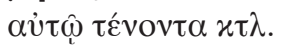

(II, 2.4.292.6-10).

La conjunción ėđeí se ve reemplazada en la koiné no literaria por ese passe-partout lingüístico en que se convierte öлоv: ${ }^{26}$

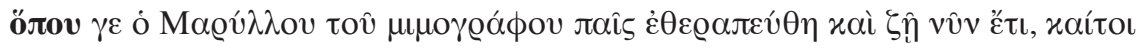

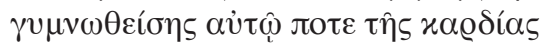

(II, 7.12.631.15-18).

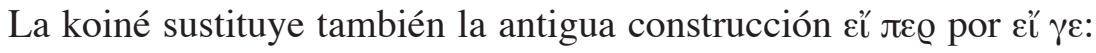

${ }^{25}$ Sin ser un listado exhaustivo, tenemos ejemplos anotados en las pp. 248, 255, 275 , $356,368,384,401,426,430,439,489,491,503,524,529,560,564,568,587,655$, 686, 695, 714 y 720 de la edición de Kühn.

${ }^{26} \mathrm{Cf}$. al respecto la sistematización de Nicholas a partir del corpus neotestamentario, Nicholas 1998, pp. 200-202. 


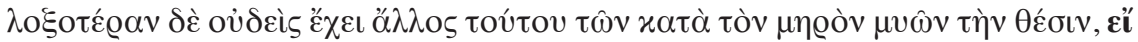

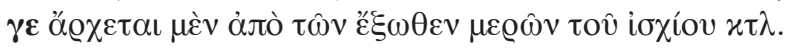

(II, 2.4.297.12-15).

Otro koinismo consiste en el empleo del pronombre غ̇xáte@os para referirse a más de dos entes:

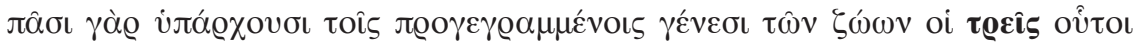

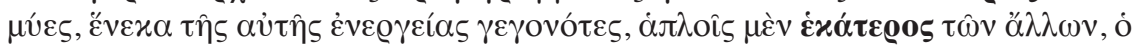

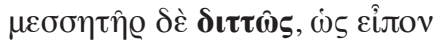

El demostrativo óde se debilita en la koiné hasta el punto de desaparecer casi por completo. Así, en el griego neotestamentario queda casi

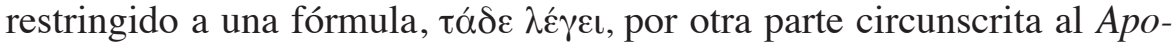
calipsis, salvo un ejemplo en los Hechos de los apóstoles. ${ }^{27}$ Fuera de esta fórmula se cuentan tan sólo dos ejemplos del empleo de óde, en la epístola de Santiago $^{28}$ y en un único pasaje de los evangelios, y no por casualidad obra del autor que escribió el griego más elaborado, el médico Lucas. ${ }^{29} \mathrm{Y}$ en Galeno López Férez advierte también la preferencia

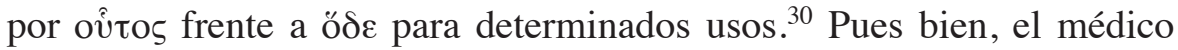

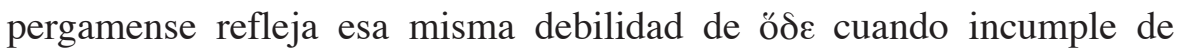
manera flagrante el precepto de utilizar dicho pronombre en la función anafórica. Esto leemos en los Procedimientos anatómicos:

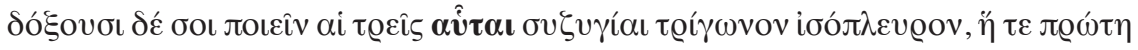

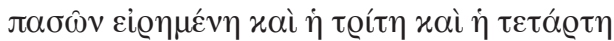

Todavía en la sintaxis pronominal, obsérvese cómo la koiné asimila

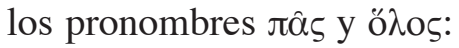

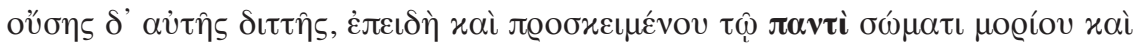

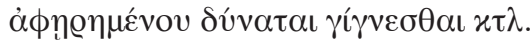

(II, 9.1.708.3-4).

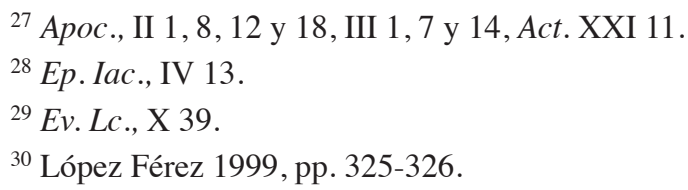


En la sintaxis de las partículas se incluyen novedades como el empleo

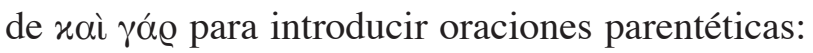

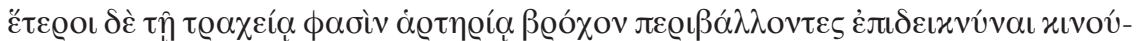

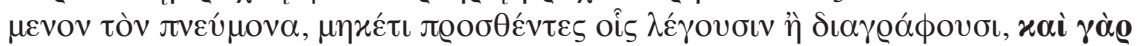

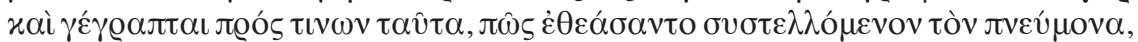

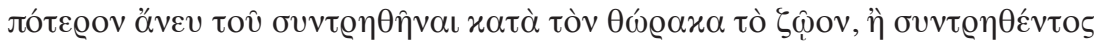

(II, 7.14.638.8-13).

También se usa con esta misma función $\mu$ غ̀v oûv:

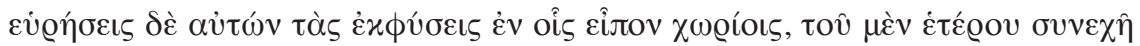

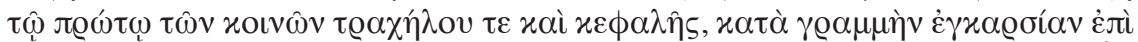

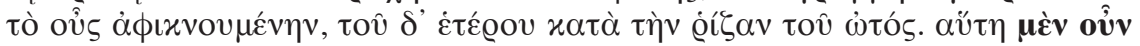

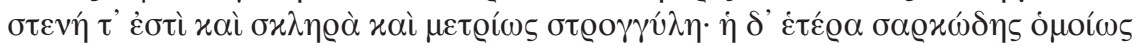

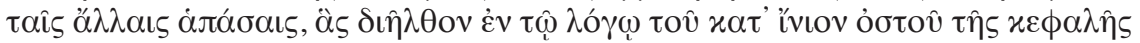

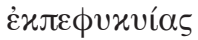

(II, 4.9.465.1-8).

Otra innovación consiste en el empleo de $\dot{\alpha} \lambda \lambda \dot{\alpha}$ con valor inceptivo e

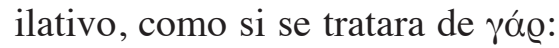

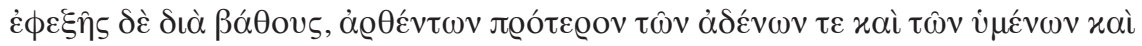

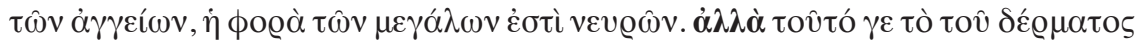

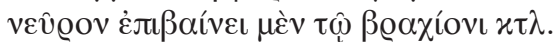

(II, 2.3.357.14-17).

a partir de aquí, y a través del interior del organismo, una vez han sido previamente extraídos los pólipos de grasa, las membranas y los vasos, tiene lugar el curso de los nervios grandes; efectivamente, este nervio de la piel remonta hasta el brazo.

\section{Veamos un nuevo ejemplo de esta construcción:}

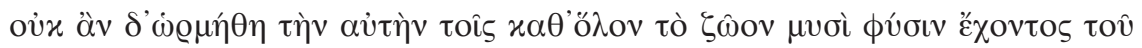

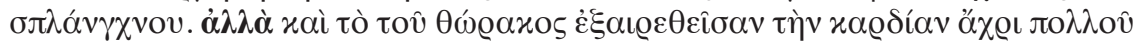

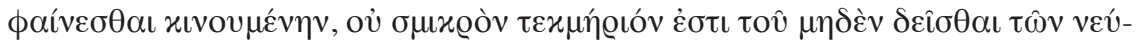

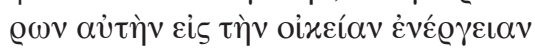

(II, 7.8.614.8-11).

no se habría movido si la víscera hubiera tenido la misma naturaleza que los músculos de todo el animal; efectivamente, el hecho de que el corazón, una vez extraído del tórax, se mueva hasta un dilatado plazo de tiempo, es una evidencia no escasa de que no necesita para nada de los nervios para la función que le es propia. 
Otro ejemplo de koinismo es el empleo de $\varkappa \alpha i ́$ to $\gamma \varepsilon$ con valor concesivo:

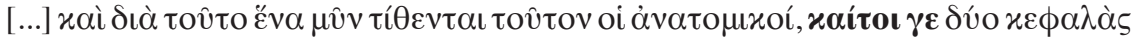

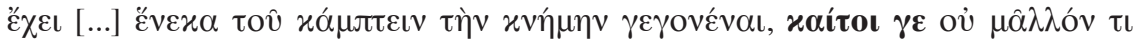

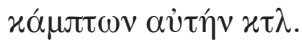

(II, 2.5.304.1-2 y 17-18).

También se inscribe entre los usos de la koiné imperial el desplazamiento de la partícula $\gamma \varepsilon$ :

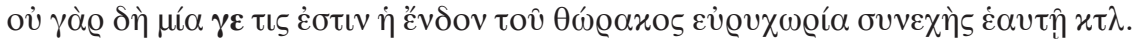

(II, 5.8.521.11-12).

Otra innovación compartida por el ático y la koiné ${ }^{31}$ consiste en el empleo de la partícula ỏ $\mu \varepsilon ́ \lambda \varepsilon ı$ :

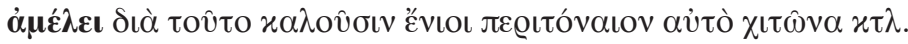

(II, 6.4.554.10).

El generoso empleo del participio concertado, tan caro a la lengua precisa y fluida de los sofistas y de Tucídides, parece próximo a un colapso morfosintáctico que lo transforme en el gerundio del griego moderno, una vez desprovisto de la función de la concordancia: ${ }^{32}$

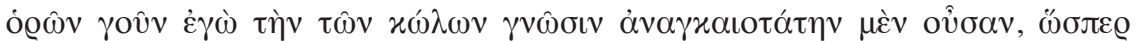

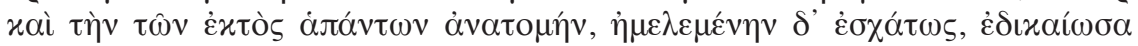

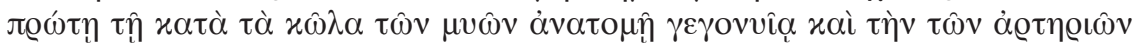

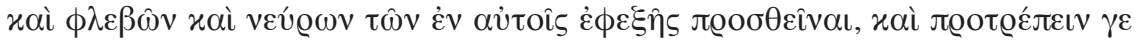

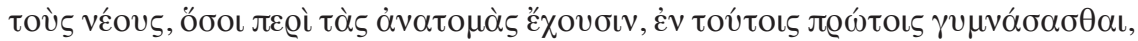

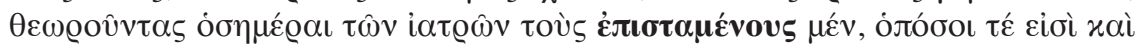

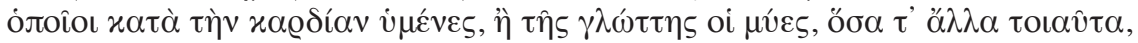

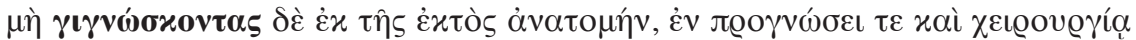

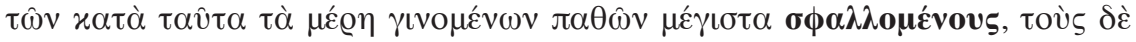

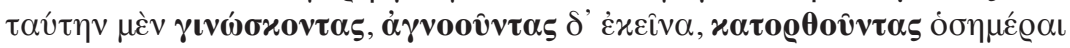

(II, 3.1.345.16-346.11).

Anotamos también como koinismo la pérdida de toda distinción entre verbos que habrían de distinguirse por su diferente valor de Aktionsart,

${ }^{31}$ López Eire 1996, pp. 104-105.

${ }^{32}$ López Eire 1984b, pp. 256-257. 


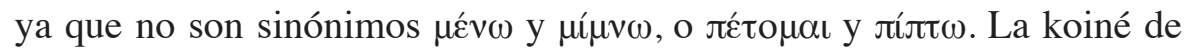
Galeno, en cambio, sí convierte en sinónimos ع̌ $\chi \omega$ e ı̌o $\chi \omega$, como si se tratara de un simple caso de uariatio:

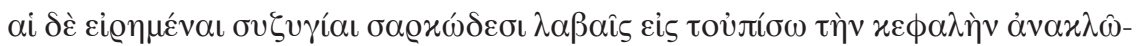

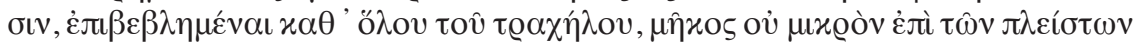

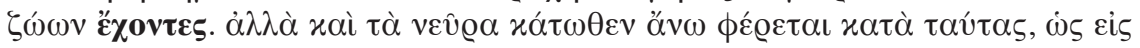

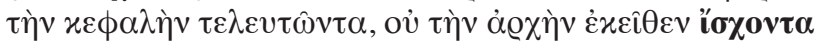

(II, 4.6.452.1-6).

También es propia de la koiné la difuminación de las diferencias entre la voz pasiva y la media a causa del paulatino declive de esta última; de ahí que Galeno las emplee en un nuevo caso de uariatio, cf. $\tau \hat{\omega} v$ ßoó $\chi \omega v$

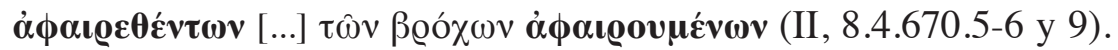

Tampoco se entiende bien, si no es por un afán de adornar el texto,

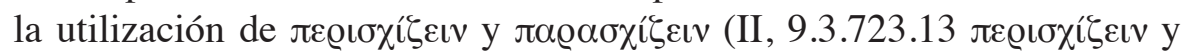

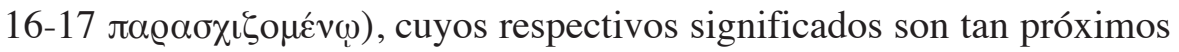
que no atinamos a diferenciarlos con claridad.

En la sintaxis de las preposiciones anotamos usos de koiné como el del empleo del adverbio $̌ ̋ \omega \theta \varepsilon v$ con genitivo:

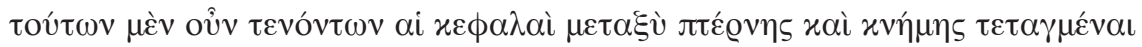

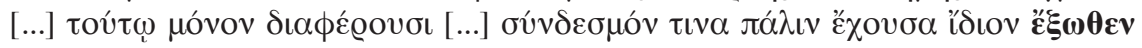
นov̂ นoเvôิ

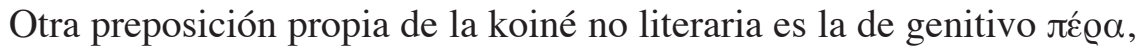
poco empleada por Galeno pero igualmente presente en nuestra obra:

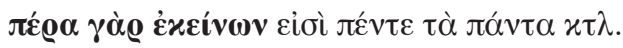

(II, 3.4.365.4).

Propia de la koiné no literaria es la preposición de genitivo vंлoxó $\omega$, que en la prosa clásica sólo utiliza con cierta frecuencia Platón: ${ }^{33}$

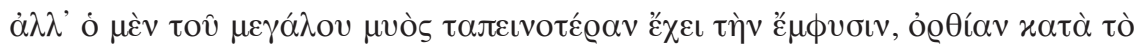

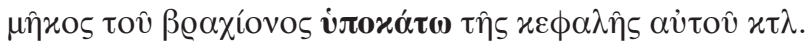

(II, 5.1.482.7-9).

${ }^{33}$ P1., Phd., 112d; Lg., 844c. 
Más infrecuente aún es el empleo de la preposición de dativo бvvór $\alpha \xi$, y que aparece en la siguiente frase:

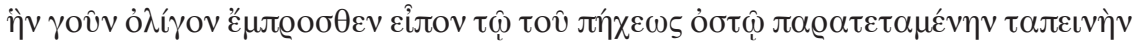

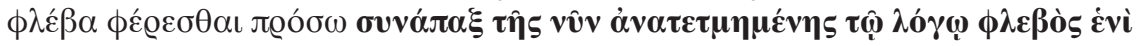

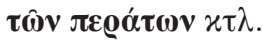

Deriva también de la koiné hablada la conflación de los adverbios $\alpha \hat{u}$ y $\pi \alpha ́ \lambda ı v$, que en la lengua clásica se distinguen bien, y que no obstante son ahora sentidos como sinónimos y colocados al unísono, a la manera de una refección:

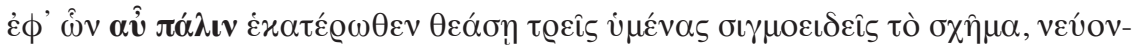

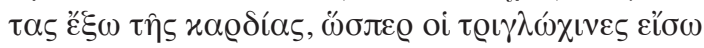

(II, 7.9.617.8-10).

Se encuadra también entre los koinismos la substitución del adverbio

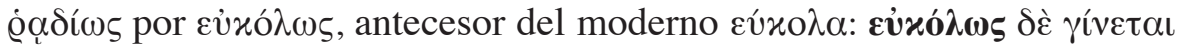

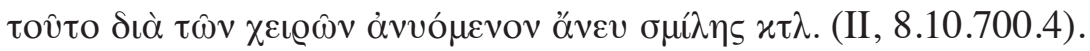

Otro koinismo consiste en el empleo del verbo ع̌@ $\pi \omega$, tomado de los

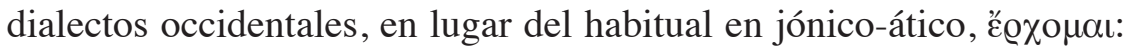

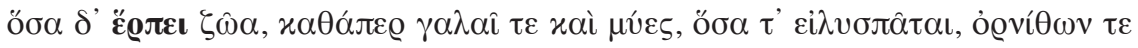

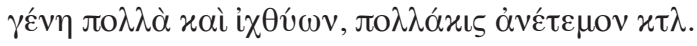

(II, 6.1.537.8-9).

Junto a esta marcada impronta de la koiné, Galeno se abre también al uso de rasgos de lengua que nada tenían que ver con la lengua hablada, sino con la tradición literaria de mayor predicamento, que era la de los autores áticos y, en todo caso, la de la ciencia jonia. A esta última pertenece, por ejemplo, tomado de la prosa científica y de la filosofia - aunque ya se encuentra en la tragedia-, el jonismo de la flexión media del verbo $\delta \varepsilon \hat{\imath}$, siempre activo en ático:

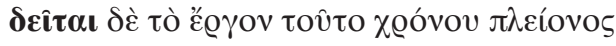

La correspondiente forma activa no parece muy frecuente, al contrario que su alternativa semántica $\chi \emptyset \eta$, al haberse convertido ambos verbos en 
sinónimos, en la práctica, una vez difuminada la antigua oposición entre obligación y conveniencia. ${ }^{34}$ Veamos un ejemplo del uso de $\delta \varepsilon \hat{:}$

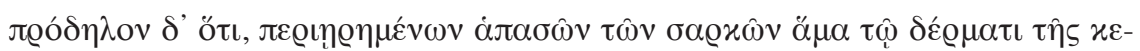

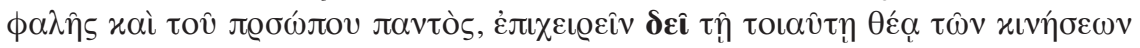
(II, 4.6.454.5-8).

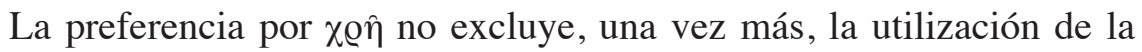
uariatio, ahora con el procedimiento de los adjetivos verbales en *-téov.

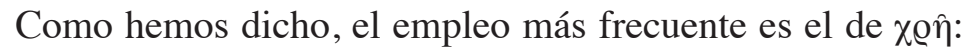

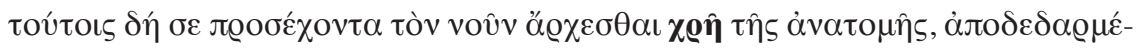

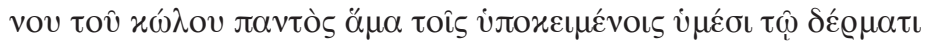

En cambio, en un pasaje similar Galeno recurre al adjetivo verbal:

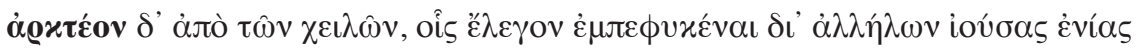

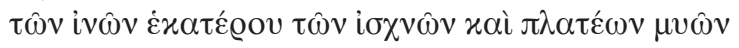

(II, 4.3.429.10-12).

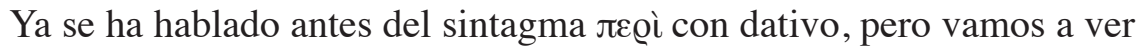
un nuevo ejemplo de esta construcción:

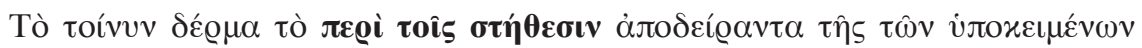

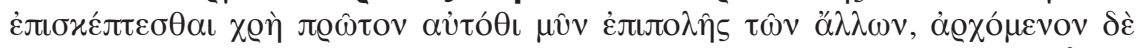

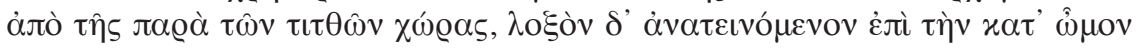

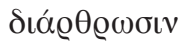

(II, 5.1.475.5-476.2).

Obsérvese, en primer lugar, que el pasaje procede del proemio del libro quinto, por lo que al tratarse de la sección que abre la exposición nuestro autor suele adornarse; y en segundo, que además del llamativo sintagma que comentamos hay que anotar el empleo del antiguo adverbio de lugar aủ ó $\theta$, atestiguado en la literatura, y que la lengua había reemplazado por el más simple aủंov̂:

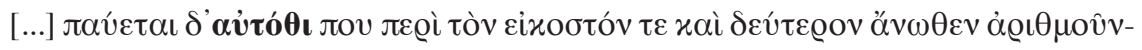

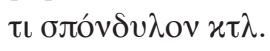

(II, 8.1.655.17-18).

\footnotetext{
${ }^{34} \mathrm{Cf}$. al respecto Benardete 1965.
} 
En este caso hablaríamos sin duda de un aticismo, pero en realidad falso, ya que el uso ático correspondía a la forma aủtov̂. Metodológicamente, pues, se trataría de un hiperaticismo.

Responde también al patrón del aticismo el empleo del optativo llamado eolio - en realidad, una formación desiderativa $-::^{35}$

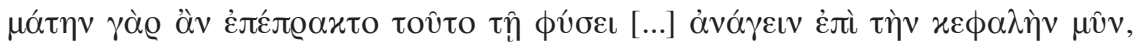

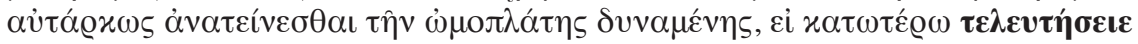
$x \tau \lambda$.

(II, 4.6.448.12-14).

Cabe describir este rasgo como un segundo ejemplo de hiperaticismo, porque esta formación de optativos de aoristo nada tiene que ver con el ático hablado, sino con un uso literario.

También pertenece a los usos de la koiné literaria el empleo como preposición de $\delta i ́ x \eta v$, atestiguado en la lírica y la tragedia clásicas y en la prosa a partir de Platón: ${ }^{36}$

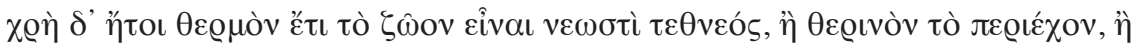

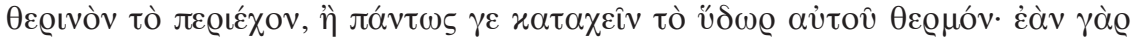

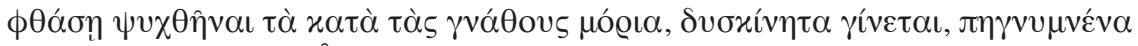

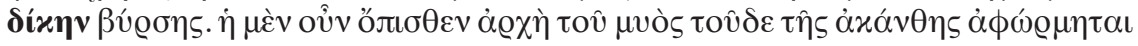

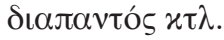

(II, 4.2.427.17-428.3).

En conclusión, nuestro autor es un perfecto conocedor de los medios para dotar a la obra del ornato propio de la más elaborada literatura; al mismo tiempo, es capaz de restringir el empleo de tales recursos y de substituirlos por otros que se relacionan con el estilo de la prosa científica y con el de la práctica de la enseñanza de la medicina. A pesar de su cuidada formación retórica y literaria, Galeno opta por una solución ecléctica - algo nada infrecuente en su práctica epistemológica-, sin tomar abierto partido ni por las soluciones aticistas ni por las de la koiné. Ahora bien, la base de su lexis ha de buscarse en la koiné no literaria, convenientemente depurada de aquellas formas y construcciones extra-

\footnotetext{
${ }^{35}$ Para la integración de este recurso morfológico en el acervo del ático literario, cf. Redondo 1986-1987, p. 95.

${ }^{36}$ Semon., frg. 11; Pi., Py., II, 84; S., frg. 569, 1; Pl., Lg., 750e.
} 
ñas a lo que sería el uso lingüístico habitual en la conversación diaria entre hablantes de una condición social media.

\section{BIBLIOGRAFÍA}

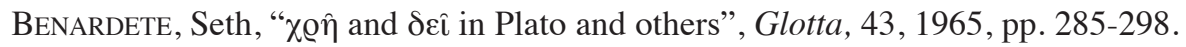
De LaCY, Philip, "Galen and the Greek Poets", GRBS, 7, 1966, pp. 259-266.

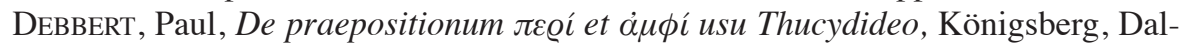
kowski, 1880.

Durling, Richard J., "Some Particles and Particle Clusters in Galen", Glotta, 66, 1988, pp. 183-189.

GoldHILl, Simon, Who needs Greek? Contests in the cultural history of Hellenism, Cambridge, Cambridge University Press, 2002.

Herbst, Wilhelm, Galeni Pergameni De atticissantium studiis testimonia collecta atque examinata pars prima seorsum expressa, Leipzig, Teubner, 1911.

Horrocks, Geoffrey, Greek. A History of the Greek Language and Its Speakers, Chichester \& Malden, Wiley \& Blackwell, 2010².

KolLeSch, Jutta, "Galen und die zweite Sophistik", in Vivian Nutton (ed.), Galen: Problems and Prospects, Londres, Wellcome Institute for the History of Medicine, 1981, pp. 1-11.

Kollesch, Jutta, "Zur Mündlichkeit hippokratischer Schriften", in Juan Antonio López Férez (ed.), Tratados hipocráticos. Estudios acerca de su contenido, forma e influencia, Madrid, UNED, 1992, pp. 335-342.

KüHN, C. G., "De anatomicis administrationibus libri novem”, Claudi Galeni opera omnia II, Leipzig, Cnobloch, 1821, pp. 125-771.

LÓPEZ EIRE, Antonio, "En torno a la lengua del Corpus Hippocraticum", Em, 52, 1984a, pp. 325-354.

LÓPEZ EIRE, Antonio, "Tucídides y la koiné", in L. A. de Cuenca et al. (edd.), Athlon: satura grammatica in honorem Francisci R. Adrados I, Madrid, Gredos, 1984b, pp. 245-262.

LóPez EIRE, Antonio, La lengua coloquial de la comedia aristofánica, Murcia, Universidad de Murcia, 1996.

LÓPEZ FÉrez, Juan Antonio, "Consideraciones sobre el léxico usado por Galeno para designar sus propias obras", in Antonio Garzya \& Jacques Jouanna (edd.), I testi medici greci. Tradizione e ecdotica, Nápoles, D’Auria, 1999, pp. 315347.

MANETTI, Daniela, "Galeno, la lingua di Ippocrate e il tempo", in Jonathan Barnes \& Jacques Jouanna (edd.), Galien et la philosophie, Vandoeuvres-Genève, Droz, 2003, pp. 171-220.

NichOLAS, Nick, The story of pu: The grammaticalisation in space and time of a Modern Greek complementiser, Melbourne, University of Melbourne, 1998.

PALM, Jonas, Über Sprache und Stil des Diodoros von Sizilien, Lund, Gleerup, 1955. 
ReDONDO, Jordi, "L' art retòrica de Xenofont I la composició de l'Agesilau”, Ítaca. Quaderns Catalans de Cultura Clàssica, 6-8, 1986-1987, pp. 83-114.

RydBeCK, Lars, Fachprosa. Vermeintliche Volkssprache und Neues Testament. Zur Beurteilung der sprachlichen Niveauunterschiede im nachklassichen Griechisch, Stockholm, Alqvist \& Wiksell, 1967.

SwaIN, Simon, "Purism without Atticism: the case of Galen", Hellenism and Empire: Language, Classicism, and Power in the Greek World AD 50-250, Oxford, Clarendon Press, 1996, pp. 56-64.

Totelin, Laurence M. V., “And to end on a poetic note: Galen's authorial strategies in the pharmacological books", Stud. Hist. Philos. Sci., 43, 2012, pp. 307-315. VAN DER EIJK, Philip J., "Towards a grammar of scientific discourse", in Egbert J. Bakker (ed.), Grammar as interpretation: Greek literature in its linguistic contexts, Leiden, Brill, 1997, pp. 77-129.

Vela Tejada, José, "Koiné y aticismo en Galeno, De antidotis: datos para un estudio lingüístico", $C F C, 19,2009$, pp. 41-61.

Vogt, Sabine, “...'er schrieb in Versen, und er tat recht daran': Lehrdichtung im Urteil Galen", in Thorsten Fögen (ed.), Antike Fachtexte: Ancient technical texts, Berlín \& Nueva York, De Gruyter, 2005, pp. 51-78.

Von Staden, Heinrich, "Galen and the 'second sophistic'", BICS, 68, 1997, pp. 3354.

Wittern-STeRZel, Renate, "Gattungen im Corpus Hippocraticum", in Wolfgang Kullmann, Jochen Althoff \& Markus Asper (edd.), Gattungen wissenschaftlicher Literatur in der Antike, Tübingen, Günther Narr, 1998, pp. 17-36. 